\title{
Transient Response of a Fluid-Filled, Thick-Walled Spherical Shell Embedded in an Elastic Medium
}

\author{
Ako Bahari ${ }^{1,2, \star}$, Gaëlle Lefeuve-Mesgouez ${ }^{2}$, Arnaud Mesgouez ${ }^{2}$, and Neil Popplewell ${ }^{1}$ \\ ${ }^{1}$ ME Department, University of Manitoba, 75A Chancellors Circle, Winnipeg, MB R3T 5V6 Canada \\ ${ }^{2}$ UMR UAPV-INRA EMMAH, Agroparc, 301 rue Baruch de Spinoza, BP 21239, 84916 AVIGNON cedex 9
}

\begin{abstract}
The paper addresses the problem of transient elastodynamics analysis of a thick-walled, fluid-filled spherical shell embedded in an elastic medium with an analytical approach. This configuration is investigated at first step for a full-space case. Different constitutive relations for the elastic medium, shell material and filling fluid can be considered, as well as different excitation sources (including $\mathrm{S} / \mathrm{P}$ wave or plane/spherical incident wave at different locations). With mapmaking visualisation, the wave propagation phenomena can be described and better understood. The methodology is going to be applied to analysis of the tunnels or other shell like structures under the effect of nearby underground explosion.
\end{abstract}

\section{Introduction}

Elasto-dynamics of an embedded spherical object or a cavity in an elastic medium has been the subject of interest since the early fifties. It is a canonical, classical problem with applications in variety of fields such as geology, civil engineering and petroleum industry. Even if they cannot always describe all the geometrical aspects of a medium, analytical developments still remain within interest since they provide results with a relatively lower cost without numerical instabilities associated with the numerical techniques. A computationally efficient approach to analyse the scattering from a spherical body as an efficient forward solver is essential within the perspective of a full wave inversion process to characterize the medium. This, also, can provide us a powerful tool towards other problems of interest such as dynamic analysis of the tunnels under the effect of the seismic waves or nearby explosion for construction purposes.

Without intending to present an exhaustive bibliographical background, a concise review is given here. Eringen [1] studied the dynamic behavior of an infinite elastic medium containing a spherical cavity subjected to a general surface traction loading. Next, Nussenzveig [2] addressed the high frequency behaviour of a totally reflecting inclusion to an incident wave using the modified Watson transformation along with other appropriate near- and far- field asymptotic in order to study the formation of different physical wave phenomena in multiple regions. Later, Norwood and Miklowitz [3] presented an analytical solution for the elastic wave propagation generated by a concentrated point source on the internal surface of the cavity as well as the impingement of a plane transient dilatational stress pulse on the external surface of the cavity. In an interesting analytical development, Datta and

^e-mail: BahariA@umanitoba.ca 
Shah [4] considered the diffraction of SH-waves originated from a point source by a rigid or fluid spherical core in which the medium outside was assumed to be spherically isotropic about its centre. Huang and Wang [5] studied the problem of transient stress wave diffraction from a spherical cavity due to a plane-step longitudinal incident wave within an elastic medium. Later on, Akkas et al. [6] developed an analytical solution for the transient radiation of the elastic waves from spherical cavity with a shell embedment excited with a non-torsional surface loading where a thin shell theory was applied to model the embedded body. Dwivedi et al. [7] solved a similar configuration as [6] in 1D for a viscoelastic soil. More recently, Schwartz et al.[8] proposed an analytical solution to the axisymmetric scattering of a cylindrical incident wave from a spherical inclusion within a viscoelastic isotropic medium in the frequency domain by applying a multi-pole expansion technique.

As it can be inferred from the pertinent literature, the problem of transient, elasto-acoustic analysis of a thick-walled, fluid-filled spherical shell embedded in an elastic medium yet to be addressed even though it looks a very classic problem. A fully transient analysis of an embedded spherical shell in an elastic medium using exact, 3D elasticity theory for the shell, with all possible sources excitation including the traction forces and plane/spherical incident waves, which is missing in the literature has been addressed in the current study. We emphasize that the methodology and procedure of solution mostly remains the same for the different constitutive laws for the solid and fluid media such as porous formation or viscous fluid as long as the Helmholtz decomposition remains applicable. The rest of the paper consists of the mathematical formulation, numerical results and conclusion remark sections.

\section{Mathematical Formulation}

A one-sided Laplace transform operator is applied to all time-dependent functions which defined as $\Lambda(s)=\mathcal{L}\{\bar{\Lambda}(t)\}=\int_{0}^{\infty} \bar{\Lambda}(t) \mathrm{e}^{-s t} \mathrm{~d} t$ and its subsequent inversion operator back to the time domain can be written as $\bar{\Lambda}(t)=$ $\mathcal{L}^{-1}\{\Lambda(s)\}=\frac{1}{2 \pi \mathrm{i}} \int_{\gamma-\mathrm{i} \infty}^{\gamma+\mathrm{i} \infty} \Lambda(s) \mathrm{e}^{-s t} \mathrm{~d} s$. As a convention, from now on, all temporal quantities in time domain come with an over-bar $(\bar{\Lambda}(t))$ and in the Laplace domain without bar $(\Lambda(s))$. For our particular application, we have made a comparison of convergence and stability of the different methods where it has been concluded that DAC algorithm with the application of a proper filter constitutes a very robust and reliable technique (for more details,

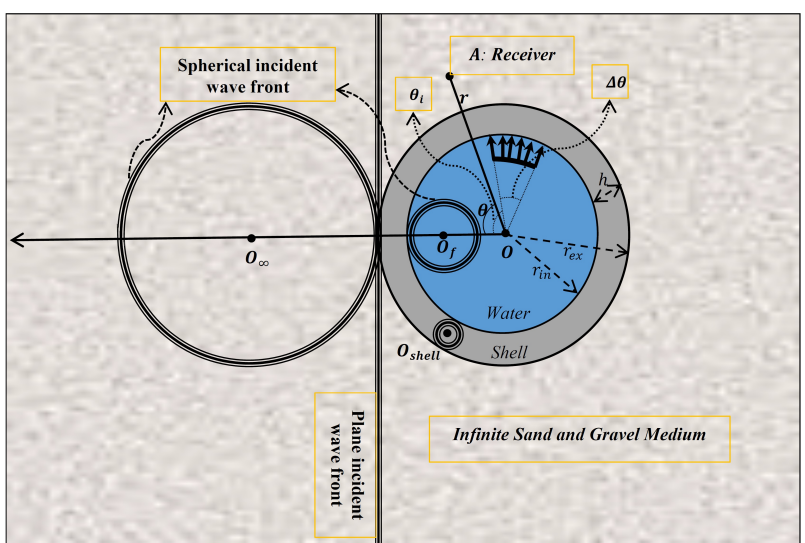

Figure 1. The problem's geometry please see [9]).

An elastic spherical shell (denoted by shell subscript) is considered in an infinite elastic formation (denoted by $\infty$ subscript) which has been filled with an ideal fluid (denoted by $f$ subscript) where the $r_{i n}^{*}, r_{e x}^{*}$ are internal and external radii of the shell as depicted in Figure1. $\mu_{k}, \lambda_{k}$ are Lamé constants and $\rho_{k}$ is density of the solid media in which the longitudinal and shear wave velocities can be obtained through $c_{p}^{(k)}=\sqrt{\frac{\lambda_{k}+2 \mu_{k}}{\rho_{k}}}$ and $c_{s}^{(k)}=\sqrt{\frac{\mu_{k}}{\rho_{k}}}$, respectively where here and from now on, $k=\{$ shell, $\infty\} . \rho_{f}, c_{f}$ stand for density and sound velocity in the internal fluid medium, respectively. The origin of the main spherical coordinate $\left(r^{*}, \theta, \phi\right)$ is chosen at the center of the shell and denoted by $O$. The point source excitation(s) in the 
infinite elastic medium $\left(O_{\infty}\right)$, shell material $\left(O_{\text {shell }}\right)$, or internal fluid medium $\left(O_{f}\right)$ has(have) the direct distance(s) of $r_{\infty}^{*}, r_{\text {shell }}^{*}$ and $r_{f}^{*}$ from $O$, respectively. The following dimensionless parameters are used throughout this paper:

$$
t=\frac{t^{*} r_{e x}^{*}}{c_{p}^{(\infty)}}, \bar{u}_{i}=\frac{\bar{u}_{i}^{*}}{r_{e x}^{*}}, v_{i}=\frac{v_{i}^{*}}{c_{p}^{(\infty)}}, r=\frac{r^{*}}{r_{e x}^{*}}, \bar{\sigma}_{i j}=\frac{\bar{\sigma}_{i j}^{*}}{\sigma_{0}}, \bar{p}=\frac{\bar{p}^{*}}{\sigma_{0}}, \alpha_{m, k}=\frac{c_{p}^{(\infty)}}{c_{m}^{(k)}}, \alpha_{f}=\frac{c_{p}^{(\infty)}}{c_{f}}
$$

in which $\sigma_{0}=\lambda_{\infty}+2 \mu_{\infty}, i, j=\{r, \theta, \varphi\}, m=\{s, p\}$ and $t, \sigma, p$, and $v$ are dimensionless time, stress, acoustic pressure perturbation and fluid particle velocity, respectively.

For sufficiently small deflections, considering the Hooke's law as the constitutive relation for the isotropic elastic medium and finally by applying the conservation law for the linear momentum in the absence of body force, the well-known Navier equation emerges as

$$
\mu \nabla^{2} \overline{\mathbf{u}}+(\lambda+\mu) \boldsymbol{\nabla}(\boldsymbol{\nabla} \cdot \overline{\mathbf{u}})=\rho \ddot{\overline{\mathbf{u}}}
$$

where the over-dot denotes the time derivatives. Based on Helmholtz decomposition $\overline{\mathbf{u}}=\boldsymbol{\nabla} \bar{\phi}+\boldsymbol{\nabla} \times \overline{\mathbf{\Psi}}$ in which the displacement vector is broken down to an irrotational vector $\boldsymbol{\nabla} \bar{\phi}$, i.e. $\boldsymbol{\nabla} \times \boldsymbol{\nabla} \bar{\phi}=0$, and a solenoidal vector $\boldsymbol{\nabla} \times \overline{\boldsymbol{\Psi}}$ means $\boldsymbol{\nabla} . \overline{\mathbf{\Psi}}=0$ (known as the gauge condition), if and only if $\nabla^{2} \bar{\phi}=\alpha_{p, k}^{2} \ddot{\bar{\phi}}$ and $\boldsymbol{\nabla} \times \boldsymbol{\nabla} \times \overline{\mathbf{\Psi}}=\alpha_{s, k}^{2} \ddot{\overline{\mathbf{\Psi}}}$ where $\bar{\phi}$ and $\overline{\boldsymbol{\Psi}}$ are the scalar longitudinal (dilatational or $P$-) wave potential and the equivoluminal (shear or $S$-) vector potential, respectively. The displacement vector elements in the spherical coordinate, using the orthogonal Hansen vectors of $(\mathbf{L}, \mathbf{M}$ and $\mathbf{N})$ reads

$$
\overline{\mathbf{u}}=\overline{\mathbf{L}}+\overline{\mathbf{M}}+\overline{\mathbf{N}}=\left(\begin{array}{c}
\bar{u}_{r} \\
\bar{u}_{\theta} \\
\bar{u}_{\varphi}
\end{array}\right)=\left(\begin{array}{c}
\frac{\partial \bar{\phi}}{\partial r}+l\left(\frac{\partial^{2}(r \bar{\chi})}{\partial r^{2}}-r \nabla^{2} \bar{\chi}\right) \\
\frac{1}{r} \frac{\partial \bar{\phi}}{\partial \theta}+\frac{1}{\sin \theta} \frac{\partial \bar{\psi}}{\partial \varphi}+l \frac{\partial^{2}(r \bar{\chi})}{r \partial \theta \partial r} \\
\frac{1}{r \sin \theta} \frac{\partial \bar{\phi}}{\partial \varphi}-\frac{\partial \bar{\psi}}{\partial \theta}+\frac{l}{r \sin \theta} \frac{\partial^{2}(r \bar{x})}{\partial \varphi \partial r}
\end{array}\right)
$$

After applying Laplace transform to the time coordinate $t$, under the zero initial condition assumption, the solution to the Navier equation is reduced to the following three scalar equations

$$
\nabla^{2} \phi=s^{2} \alpha_{p, k}^{2} \phi, \quad \nabla^{2} \psi=s^{2} \alpha_{s, k}^{2} \psi, \quad \nabla^{2} \chi=s^{2} \alpha_{s, k}^{2} \chi .
$$

Using the method of separation of variables, three dimensional solutions of the above mentioned equations, by ensuring a bounded value for the potentials as $r \rightarrow \infty$ in the both elastic media may be expressed as

$$
\left(\begin{array}{c}
\phi^{(\infty)} \\
\psi^{(\infty)} \\
\chi^{(\infty)} \\
\phi^{(\text {shell })} \\
\psi^{(\text {shell })} \\
\chi^{(\text {shell })}
\end{array}\right)(r, \theta, \varphi, s)=\sum_{n, m}^{\infty}\left(\begin{array}{c}
A_{n}^{m} k_{n}\left(r s \alpha_{p, \infty}\right) \\
B_{n}^{m} k_{n}\left(r s \alpha_{s, \infty}\right) \\
C_{n}^{m} k_{n}\left(r s \alpha_{s, \infty}\right) \\
D_{n}^{m} i_{n}\left(r s \alpha_{p, \text { shell }}\right)+E_{n}^{m} k_{n}\left(r s \alpha_{p, \text { shell }}\right) \\
F_{n}^{m} i_{n}\left(r s \alpha_{s, \text { shell }}\right)+G_{n}^{m} k_{n}\left(r s \alpha_{s, \text { shell }}\right) \\
H_{n}^{m} i_{n}\left(r s \alpha_{s, \text { shell }}\right)+I_{n}^{m} k_{n}\left(r s \alpha_{s, \text { shell }}\right)
\end{array}\right) \tilde{Y}_{n}^{m}(\theta, \varphi)
$$

where $\sum_{n, m}^{\infty} \equiv \sum_{n=0}^{\infty} \sum_{m=-n}^{+n}, \tilde{\mathrm{Y}}_{n}^{m}(\theta, \varphi)=\sqrt{\frac{(n-m) !(2 m+1)}{4 \pi(n+m) !}} P_{n}^{m}(\cos \theta) \mathrm{e}^{\mathrm{i} m \varphi}$ in which $P_{n}^{m}$ is the associated Legendre function. $i_{n}$ and $k_{n}$ are the modified spherical Bessel functions of first and second kind, respectively. Using the Hansen vectors

$$
\left(\begin{array}{c}
\mathbf{u}_{\infty} \\
\mathbf{u}_{\text {shell }}
\end{array}\right)=\sum_{n, m}^{\infty}\left(\begin{array}{c}
A_{n}^{m}{ }_{k}^{\infty} \mathbf{L}_{n}^{m}+B_{n}^{m}{ }_{k}^{\infty} \mathbf{M}_{n}^{m}+C_{n}^{m}{ }_{k}^{\infty} \mathbf{N}_{n}^{m} \\
D_{n}^{m}{ }_{i}^{s h} \mathbf{L}_{n}^{m}+E_{n}^{m}{ }_{k}^{s h} \mathbf{L}_{n}^{m}+F_{n}^{m}{ }_{i} \mathbf{M}_{n}^{m}+G_{n}^{m}{ }_{k} \mathbf{M}_{n}^{m}+H_{n}^{m}{ }_{i} \mathbf{N}_{n}^{m}+I_{n}^{m}{ }_{k}^{s h} \mathbf{N}_{n}^{m}
\end{array}\right),
$$


The displacement vectors and consequently the stress tensor of the solid media are derived.

The conversion of mass and linear momentum in a stagnant fluid medium, for an acoustic phenomenon, in the absence of body force reads $\rho_{f} \overline{\mathbf{v}}_{, t}+\nabla \bar{p}=0$. Using the acoustic constitutive relation of $p=c_{f} \rho_{f}^{\prime}$ and aforementioned conservation rules, $\rho_{f}^{\prime}$ and $\mathbf{v}$ can be omitted. After applying the Laplace transform with respect to the time domain to the resulting acoustic wave equation, its subsequent solution, in terms of spherical harmonics, becomes

$$
\nabla^{2} p=\alpha_{f}^{2} s^{2} p \Rightarrow p(r, \theta, \varphi, s)=\sum_{n, m}^{\infty} J_{n}^{m} i_{n}\left(\alpha_{f} r s\right)
$$

where $A_{n}^{m}=A_{n}^{m}(s, n, m)$ through $J_{n}^{m}=J_{n}^{m}(s, n, m)$ are the unknown coefficients to be determined after enforcing the complete continuity of the stress and displacements at fluid-solid and solid-solid interfaces.

A general case of the traction forces is explained first and followed by a point source inside the fluid. Later the point sources inside the shell material is defined and then the point source and incident plane waves are considered finally. In all of the incident wave cases, $f(t)$ is an arbitrary temporal function of the excitation source. Also, the origin of the time, $t=0$, is set at the moment that the wave front touches one of the traction boundaries, i.e. inner or outer shell surface, since the domain solution prior this point does not provide any information of interest. In the time domain, all incident waves can be expressed in the following general form as

$$
\bar{p}^{(i n c)} \text { or } \bar{\phi}^{(i n c)} \text { or } \bar{\psi}^{(i n c)} \text { or } \bar{\chi}^{(i n c)}(r, \theta, t)=r^{\prime \prime} \bar{f}(\tau) \mathcal{H}(\tau)
$$

where $\mathcal{H}(t)$ is the Heaviside function and $r^{\prime \prime}=r^{\prime \prime}(r, \theta, t), \tau=\tau(r, \theta, t)$ are defined in each case. After applying the Laplace transform, the classical translational addition theorem is applied in order to express the excitation sources in terms of the spherical harmonics with respect to the main coordinate located at $O$ (in Figure1); this takes the following general form of

$$
\left(p^{(i n c)} ; \phi^{(i n c)} ; \psi^{(i n c)} ; \chi^{(i n c)}\right)(r, \theta, \varphi, s)=\sum_{n, m}^{\infty}\left(p_{n m}^{(i n c)} ; \phi_{n m}^{(i n c)} ; \psi_{n m}^{(i n c)} \chi_{n m}^{(i n c)}\right)(r, s, n, m) \tilde{\mathrm{Y}}_{n}^{m}(\theta, \varphi)
$$

where $p_{n}^{(i n c)}, \phi_{n}^{(i n c)}, \psi_{n}^{(i n c)}$ and $\chi_{n}^{(i n c)}(r, s)$ are adapted for each case.

\section{Numerical results}

In all case studies, a Rikcer pulse is adapted as $\bar{f}(t)=\left(1-2\left(\frac{t-t_{0}}{t_{\omega}}\right)^{2}\right) \mathrm{e}^{-\left(\frac{t-t_{0}}{t_{\omega}}\right)^{2}}$, in which $t_{0}=0.5, t_{\omega}=$ 0.1 are chosen as non-dimensional parameters. A special visualization technique is used to map the total field solution in discrete time snapshots. Each snapshot depicts radial stress $\left(\bar{\sigma}_{r r}(r, \theta, t)\right)$ solid media and pressure $(\bar{p}(r, \theta, t))$ in normalized form in a way that always the grey color associated with the zero value remains the same in all snapshots. Considering a typical dense sand for the elastic formation, a polyurethane shell and water as the internal fluid; the non-dimensional celerity and density ratios are $\alpha_{P}^{\text {Shell }}=1.29, \alpha_{S}^{\text {Shell }}=46, \alpha_{P}^{\infty}=1, \alpha_{S}^{\infty}=0.5, \alpha_{f}=0.54, \rho_{\infty}=1, \rho_{\text {shell }}=$ $0.6, \rho_{f}=1.2$ and the shell thickness is assigned to be $h\left(=r_{i n}-r_{e x}\right)=0.2$.

A P-wave source point is considered at $r=3$ which touches the shell outer surface for the first time at $t=2$. Incident wave is excluded so the only scattered wave in the external medium is seen as shown in Figure 2. As soon as the interaction begins, the refraction of the P-and S- waves as well as specular reflection in both compressional and shear waves can be seen although the incident wave 


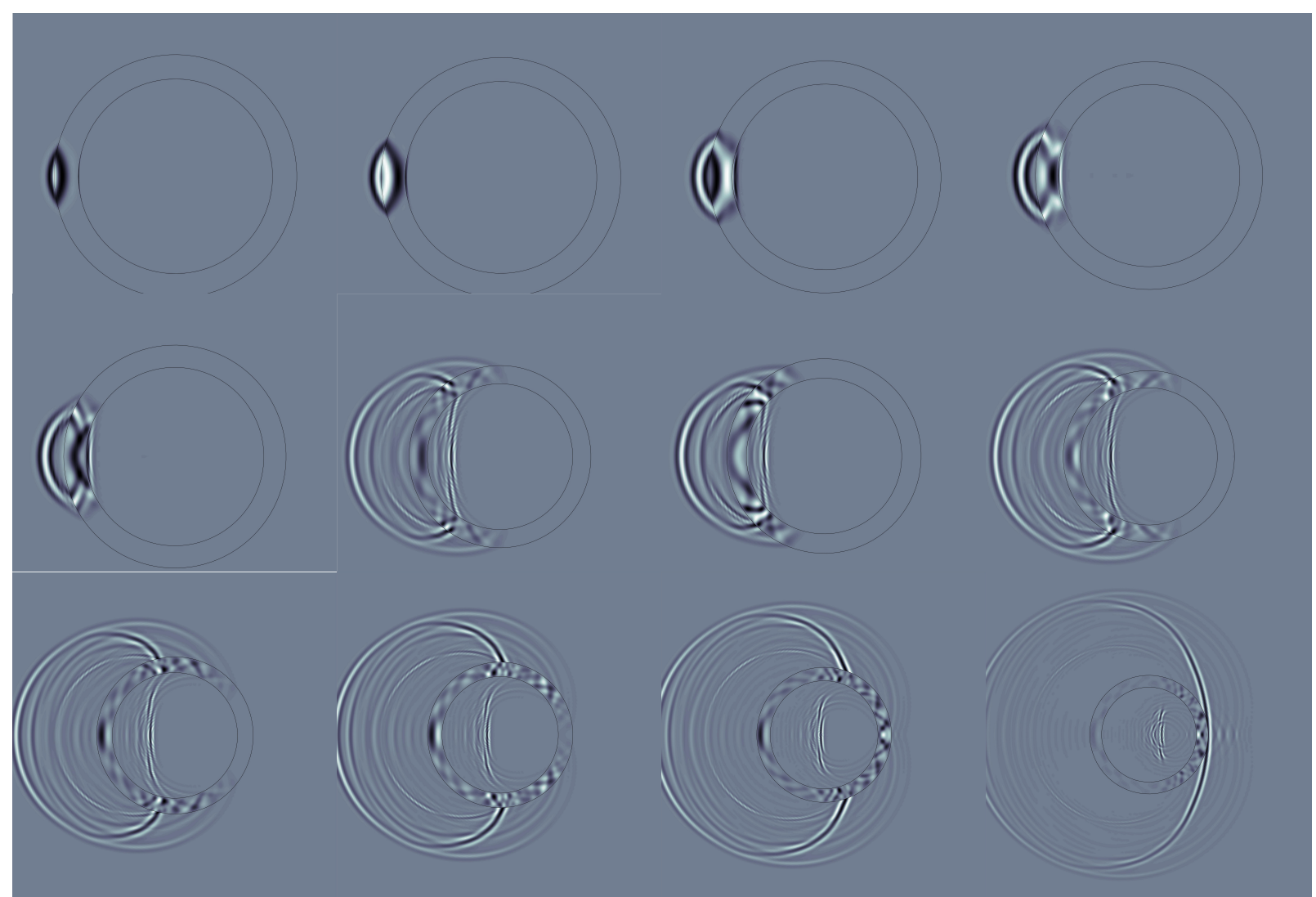

Figure 2. Pressure and radial stress resulted from an elastic, incident P-wave coming from a point located in the external elastic medium, for different time values.

is a pure longitudinal wave. This phenomenon happens due to the mode coupling at the interface as the boundary conditions are enforced. Since the celerity of the P- and S- wave is higher in the shell material, the wave fronts propagates faster in the shell. Next, as the the wave front touches the internal surface, another mode conversion happens at the reflection. Even though in this case, only a compressional wave transmits to the fluid medium since the ideal fluid can not sustain a shear wave. As time passes by, each P-/S- wave travels the shell's thickness and reaches the either interfaces, the mode conversion happens. It means that each $\mathrm{S}-/ \mathrm{P}$ - wave transmits and reflects in both forms. When incident $\mathrm{P}$-wave passes half through the shell, i.e. $t>1$, as demonstrated in Figure 3, the formation of the internal wave triple points within the fluid starts which leads to a strong focusing phenomenon that can amplify the initial refracted waves significantly by up to four times fold. This point can provide the vital information in the design and structural integrity as a whole.

In Figure 3, unlike the previous case study, the source of the incident wave is located within the internal fluid at $r=0.4$. The incident term is not omitted, so the internal pressure is a representation of the total pressure. As incident pressure reaches the inner surface, a specular pressure wave reflects back to the water, and it stays only wave in addition to the incident wave. Due to the mode conversion at the water-shell interface, P- and S- waves are created in the shell, and as these get to the outer interface, other sets of mode conversion happen as well as a part of the wave which is reflected as P-/S- waves into the shell from either interfaces. When these waves meet the either of interfaces, the same scenario happens over and over again. Because of the circular reflector shape of the shell's inner surface, a couple of triple points starts forming as time progresses in the second raw of Figure 3. These triple points, later conclude into a set of focusing amplification in the pressure and stress levels. 


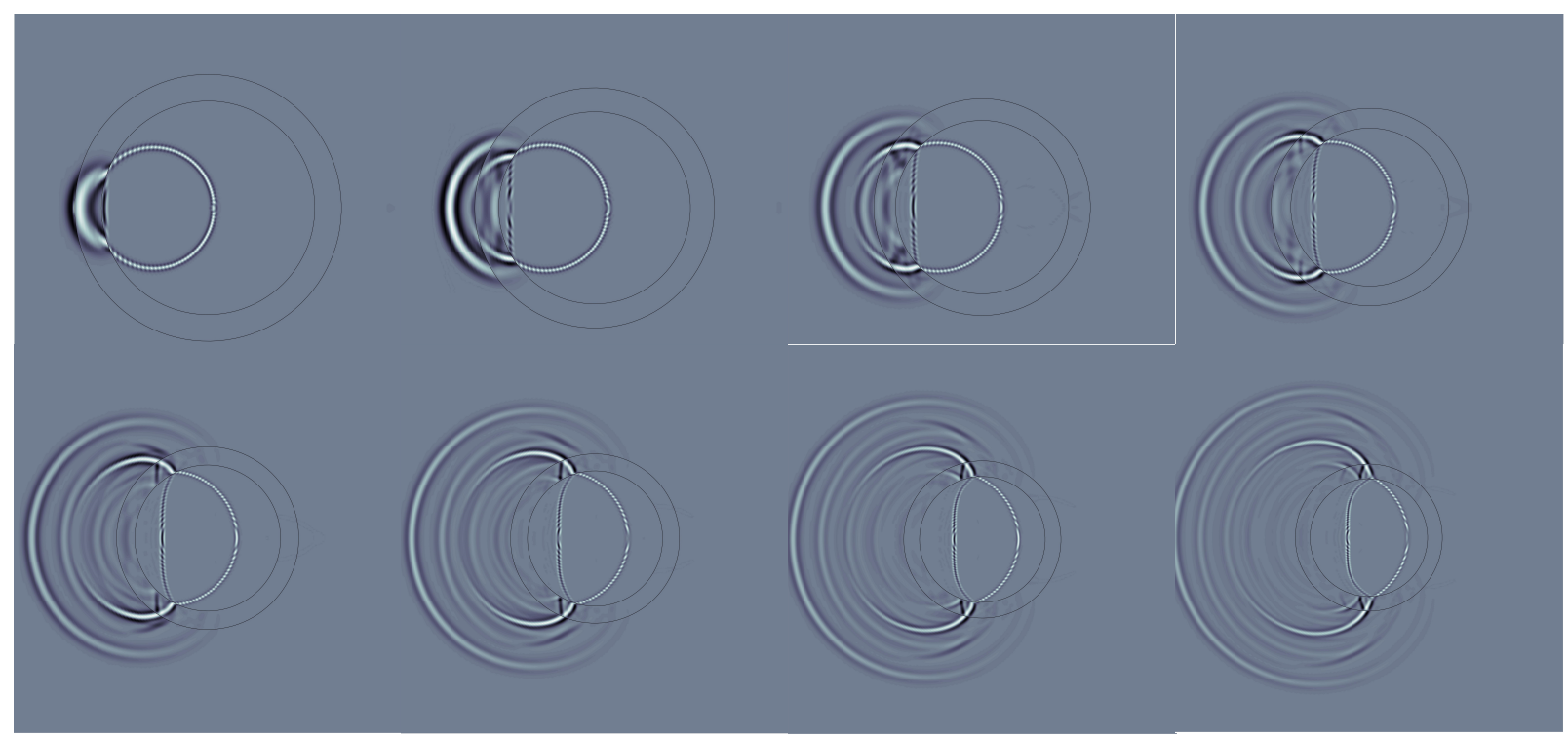

Figure 3. Pressure and radial stress resulted from an internal incident pressure P-wave, for different time values.

\section{Conclusion and Perspectives}

So far, the transient problem of a fluid-filled, hollow sphere in an infinite elastic medium under different excitation sources has been solved with an analytic approach. Different wave phenomena, such as the formation of triple points and focusing mechanism, have been observed. These data are crucial for the design and structural integrity as well as underground imaging of the spherical inclusions and anomalies. In the future, other constitutive laws for the solid media and filling fluid are going to be considered including the porosity and damping effects. In addition, similar developments will be oriented also to deal with the LSBB geometry configuration of one or several tunnels included into a multilayered ground model under the effect of the incident excitation. All these achievements fit into a long-term pattern that aims at identifying and quantifying the main influential mechanical parameters to characterize the LSBB ground from a mechanical point of view. The tunneling programmed soon at the LSBB constitutes a great opportunity to get experimental results to construct an inversion strategy.

\section{References}

[1] Eringen, A. C., Q J Mech Appl Math, 10, 257-270 (1957)

[2] Nussenzveig, H. M., Annals Physics 34, 23-95 (1965)

[3] Norwood, F. R., and Miklowitz, J Appl Mech, 34, 735-744 (1967)

[4] Datta, S. K., and Shah, A. H., Geophysical Journal Int 21, 33-46 (1970)

[5] Huang, H., and Wang, Y., J Appl Mech, 39, 1002-1004 (1972)

[6] Akkas, N., Zakout, U., and Tupholme, G., Acta Mechanica 142, 1-11 (2000)

[7] Dwivedi, J., Singh, V., and Lal, R. K., ISRN Mech Eng 2013, 1-10 (2013).

[8] Schwartz, B. L., Liu, Y., Royston, T. J., and Magin, R. L., J Sound Vib, 364, 222-233 (2016)

[9] Laverty, R., and Gazonas, G.A., Int J of Comp Meth, 3.01, 57-69 (2006) 